\title{
Enabling Kinetically-unfavorable Phase Transformation Mechanisms with Plasmonics
}

\author{
Katherine Sytwu, Michal Vadai, Fariah Hayee, Alan Dai, Jefferson Dixon and Jennifer Dionne
}

Stanford University, Stanford, California, United States

Palladium is a commonly used catalyst in hydrogenation reactions due to its affinity to adsorb hydrogen, but the subsequent absorption of hydrogen, or the phase transformation into palladium hydride, can affect the catalytic activity and/or selectivity [1]. Currently, the formation/dissolution of palladium hydride is controlled via setting macroscopic parameters such as temperature and chemical potential (i.e. hydrogen pressure). Local surface plasmon resonances (LSPRs) offer an alternative, optical route to control the palladium hydride phase transition due to the LSPR decay products of light, hot carriers, and heat [2]. Additionally, LSPRs support non-uniform electromagnetic enhancement profiles that could provide subparticle spatial control of the phase transition. However, most techniques to track nanoparticle phase transitions lack the ability to identify differences in transient behavior at the sub-particle level.

Here, we utilize in-situ environmental transmission electron microscopy combined with optical excitation to analyze how LSPRs modify the dehydrogenation phase transition of Pd nanorods. Taking advantage of the $\sim 3.5 \%$ decrease in Pd's lattice parameter upon dehydrogenation, we use displaced aperture dark field imaging (Figure 1a) to track the differences in nucleation site of individual nanoparticles in real time as we vary external parameters like optical illumination and hydrogen pressure. First, we find that without illumination, these colloidally synthesized, 400-600nm Pd nanorods undergo their phase transition starting from both of the nanorod tips, as seen in Figure 1b. In these dark field images, signal represents the hydrogenated beta phase while the lack of signal represents the dehydrogenated alpha phase

Next, to identify the role of the LSPR, we design a Au-Pd crossed-bar structure which utilizes the strong visible-frequency LSPR response of Au. We lithographically pattern 60-90nm wide Au nanobars onto a $\mathrm{Si}_{3} \mathrm{~N}_{4} \mathrm{TEM}$ grid, then atomic layer deposit $2 \mathrm{~nm}$ of $\mathrm{SiO}_{2}$ to create a spacer layer, followed by dropcasting colloidally synthesized Pd nanorods. This geometry supports a gap plasmon mode between the Au nanobar and the Pd nanorod, creating a localized area of strong electromagnetic enhancement at the middle of the Pd nanorod, spatially separated from the favorable tip nucleation sites (Figure 2a-b). Under illumination at the plasmon resonance, $\mathrm{Pd}$ nanorods that are coupled to the plasmonic Au antenna demonstrate either nucleation of the dehydrogenated phase from only one tip, or nucleation from the middle of the nanorod near the electromagnetic hot spot (Figure 2c). Wavelength-dependent illumination studies confirm that this middle-nucleation behavior follows the plasmonic response of the Au nanobar and is driven by the external illumination. Furthermore, we track the nucleation behavior of over 20 Au-Pd pairs under resonant illumination as we vary the surrounding hydrogen pressure. About half of the particles show single-tip nucleation, while the other half show middle nucleation but only at lower hydrogen pressures, indicating that the middle nucleation phase transition mechanism is less energetically favorable than tip nucleation and only accessible via plasmonic excitation.

Our results demonstrate how optical illumination can not only increase kinetics but also enable new phase transformation mechanisms. Our proof-of-concept design allows for direct, sub-particle visualization of the LSPR's effect on phase transformations, and provides a foundation for site-selective and productspecific reactions via optical control. 


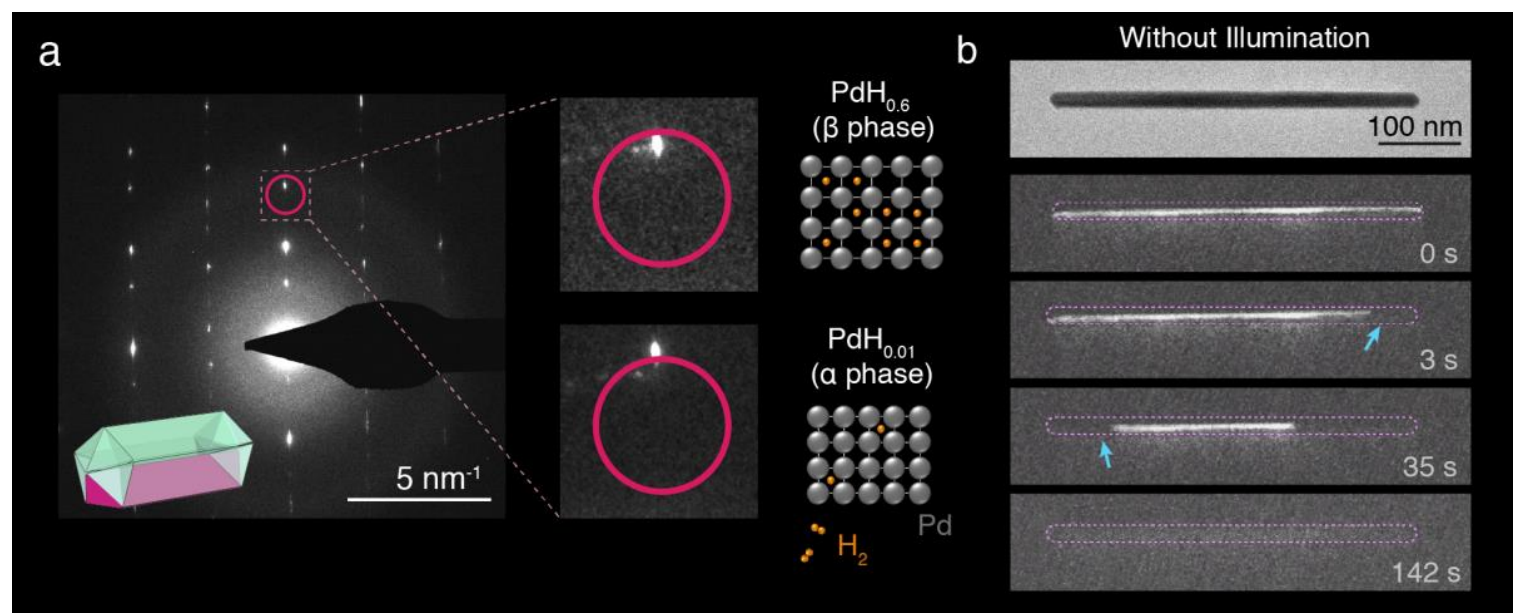

Figure 1. (a) Diffraction pattern of a colloidally synthesized pentatwinned Pd nanorod. The pink circle indicates the position of the objective aperture such that the subsequent dark field image captures the hydrogenation state of the bottom crystallite. Insets show how the diffraction point moves out of the aperture as the particle goes from its hydrogenated, lattice-expanded beta-phase to its dehydrogenated alpha phase. (b) TEM image and time series of dark field images of an isolated Pd nanorod as it dehydrogenates from beta to alpha phase, with a drawn outline as a guide-to-the-eye. Note that the dehydrogenated phase (indicated by lack of signal) comes in from both tips of the nanorod.

a

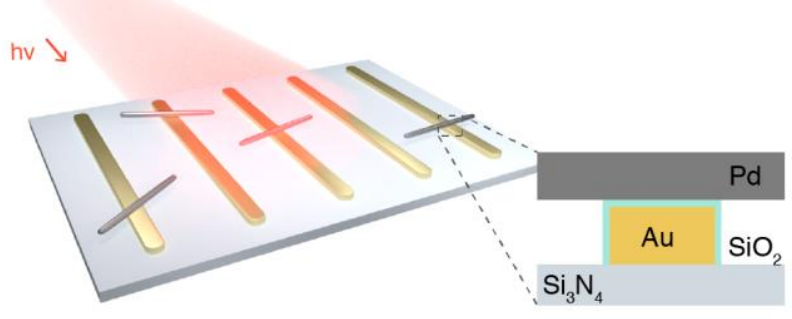

b

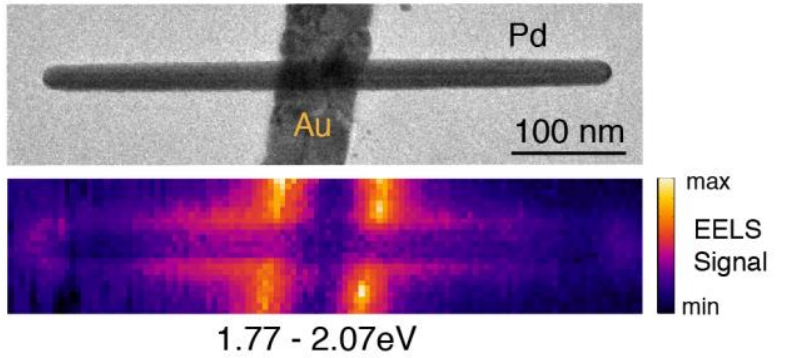

C
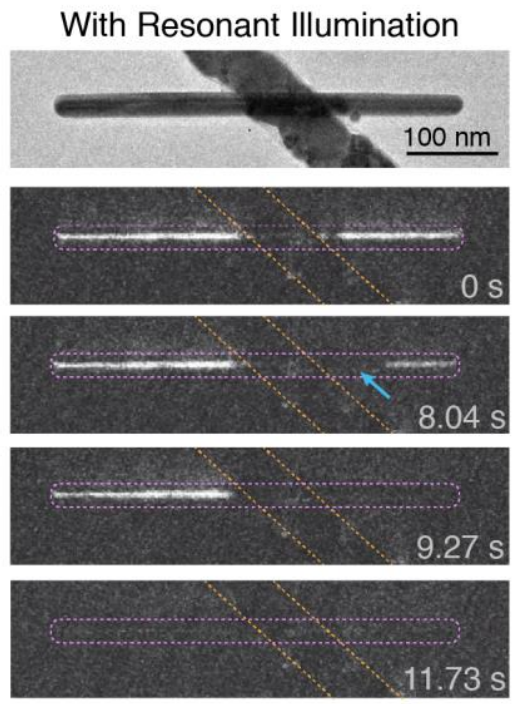

Figure 2. (a) Schematic depicting the illuminated Au-Pd crossed bar system, which consists of a colloidally synthesized Pd nanorod crossed with a plasmonic Au nanobar. Inset shows a cross-sectional view. (b) TEM image of one such Au-Pd pair, and its corresponding energy-filtered electron-energy loss spectroscopy (EELS) map from 1.77-2.07eV, demonstrating the LSPR response. (c) TEM image and time series of dark field images of a Au-Pd pair, showing the phase transformation mechanism under resonant illumination with a drawn outline as a guide-to-the-eye. The nucleation of the dehydrogenated phase is now at the middle of the Pd nanorod, near the electromagnetic enhancement.

\section{References}

[1] Billeter, E., Terreni, J., \& Borgschulte, A. ChemPhysChem 20 (2019) 1398-1403.

[2] Vadai, M., Angell, D.K., Hayee, F., Sytwu, K., \& Dionne, J. A. Nature Communications 9 (2018) 1-8. 